\title{
Causas de la restricción eléctrica en el primer franquismo: una aportación desde la historia empresarial
}

\section{Causes of electrical restriction in the first years of Franco era: a contribution from business history}

\author{
PABLO DÍAZ MORLÁN \\ Universidad de Alicante \\ ELENA SAN ROMÁN \\ Universidad Complutense de Madrid
}

\begin{abstract}
RESUMEN
Nuestro trabajo tiene dos objetivos. El primero y más importante es proponer una nueva causa de las restricciones eléctricas sufridas durante el primer franquismo, consistente en las implicaciones que tuvo para el sector eléctrico el pacto firmado en 1934-36 entre Saltos del Duero y las empresas agrupadas en lo que se dio en llamar grupo hidroeléctrico. Por este convenio, Saltos del Duero se erigió en productor dominante y el grupo hidroeléctrico en distribuidor en dos zonas de España, la centro y la norte-noroeste, lo que convirtió a Duero en el principal decisor en materia de nuevas construcciones. Dicho pacto implicó un radical conflicto de intereses entre Duero, a quien le convenía moderar sus inversiones hasta tener garantizada la colocación de la nueva producción, y el grupo hidroeléctrico, deseoso de disponer de energía abundante. Los gestores de Duero, dominados por los problemas y la mentalidad de los años treinta, cuando el problema era la falta de demanda, dificultaron la respuesta de la oferta. El segundo objetivo, por último, es mostrar nuestras dudas acerca de

la relevancia que tuvo la congelación de las tarifas en las decisiones de inversión.
\end{abstract}

PALABRAS CLAVE: Restricción eléctrica, franquismo, pacto de 1934-36, Saltos del Duero

Códigos JEL: N74

\section{ABSTRACT}

Our work has two goals. The first and most important is to propose a new cause of electrical restric-tions suffered during the forties, consisting of the pact signed in 1934-36 between Saltos del Duero and hydroelectric group. Because of this agreement, Saltos del Duero was converted in the dominant producer in two areas of Spain, the centre and northnorthwest, which turned Duero in the main decision-maker in terms of new construction. Such a pact meant a radical conflict of interests between Duero, who wanted to moderate their investments, and hydroelectric group, eager to have abundant energy. The managers of Duero, dominated by the problems and the mentality of the thirties, when the problem was lack of demand, hindered the supply response. Finally, the second goal is to show our doubts about the relevance of low tariffs in the investment decisions.

KEY WORDS: Electrical restriction, Franco's Regime, 1934-36 agreement, Saltos del Duero

JEL Codes: N74 


\section{Introducción ${ }^{1}$}

$\mathrm{U}$

no de los problemas más graves y conocidos de la historia económica del primer franquismo fue la "penuria energética"2. La insuficiencia de la oferta frente a la demanda resultó especialmente intensa en el terreno del petróleo y en el de la electricidad. La escasez de petróleo era, en buena medida, una cuestión de política exterior que se solucionó cuando se suavizaron las sanciones y las estrecheces provocadas por la II Guerra Mundial. Sin embargo, la incapacidad de atender la demanda eléctrica, que se alargó hasta 1957, respondía a otros motivos, bastante complejos ${ }^{3}$.

Han sido cinco las causas principales que se han apuntado para explicar la restricción eléctrica de la posguerra. En primer lugar, es sabido que las autoridades del régimen franquista recurrieron a la "pertinaz sequía" para dar explicación al problema eléctrico. Sin negar el incuestionable argumento meteorológico y su influencia, los empresarios de la época aportaron otros tres de mayor calado: las dificultades para importar material eléctrico, la ausencia de "unidad de explotación", es decir, de una red única, y la congelación de las tarifas. El asunto de la red única era un problema real que los empresarios particulares utilizaron como argumento para crear Unidad Eléctrica S.A. (UNESA) y defenderse de la amenaza que suponía, a la altura de 1944, el desembarco del Instituto Nacional de Industria (INI) en el sector eléctrico a través de la creación de la Empresa Nacional de Electricidad (ENDESA) ${ }^{4}$. Respecto a las tarifas, su ordenamiento se remontaba a un Real decreto de 1924 que fue confirmado en 1933 y no se revisó hasta 1951. Su inmovilidad ha sido una de las razones más frecuentemente esgrimidas desde los años cuarenta hasta la actualidad a la hora de explicar la escasez eléctrica y una de las que se ha ponderado como de mayor importancia: con precios fijos desde antes de la guerra civil y en una coyuntura de inflación galopante es lógico que las empresas evitaran nuevas inversiones,

[Fecha de recepción del original, abril 2008 . Versión definitiva de septiembre 2008]

1 Este trabajo se ha beneficiado de los comentarios y sugerencias que nos hicieron los asistentes a dos eventos: las Jornadas de Historia de la Electricidad que se celebraron en la Universidad de Barcelona los días 15 y 16 de mayo de 2008, y la sesión n 6 del IX Congreso Internacional de la Asociación Española de Historia Económica que, con el título de Tarifas y regulación de la industria eléctrica, tuvo lugar el 11 de septiembre del mismo año. Queremos dejar constancia de nuestro agradecimiento a ellos y a los evaluadores anónimos de Investigaciones de Historia Económica.

2 Sudrià (1994), p. 330 y (2007), vol I, pp. 21-22. Pueden consultarse buenos resúmenes en Sudrià (1990); Barciela, López, Melgarejo y Miranda (2001) y Pueyo (2007).

3 Los primeros apagones por incapacidad productiva se produjeron en 1944. La peor época correspondió a los años 1944-1950 y, en concreto, los peores años fueron 1945 y 1949. Sudrià calcula que en estas últimas fechas citadas la restricción superó el 20 por ciento de la demanda. Sudrià (2001), p. 15.

4 UNESA nació para regular el sector y establecer una red de interconexiones. Véase Gómez Mendoza (2000), pp. 69-70; (2007), vol I, pp. 443-444. 
que el consumo se disparara y que la ausencia de inversión se convirtiera en un auténtico cuello de botella 5 .

El propio aumento del consumo constituye la quinta explicación al problema energético de la posguerra: mientras que en los años treinta lo habitual había sido el exceso de oferta de electricidad, en la posguerra la situación sufrió un vuelco que, al menos hasta 1942, no parecía alarmar a muchos. Gómez Mendoza ha demostrado que las autoridades franquistas no se plantearon la escasez de electricidad como problema hasta la fecha citada y ello le ha permitido afirmar que antes se produjo una "apatía oficial hacia la cuestiones eléctricas" ${ }^{\prime \prime}$. En las páginas siguientes comprobaremos que la principal empresa productora de electricidad, Saltos del Duero, manifestó en la inmediata posguerra su oposición a aumentar su capacidad generadora precisamente por el "fantasma" del exceso de producción. De hecho, entre 1935 y 1943 el consumo de electricidad aumentó todavía de forma moderada, un 10 por ciento, y este incremento fue satisfecho sin dificultad con el margen aún no utilizado del sistema hidroeléctrico y con la intensificación en el uso de la potencia térmica instalada7.

La reciente investigación que hemos realizado en los archivos de algunas de las más importantes empresas del sector eléctrico nos ha permitido tomar conciencia de una nueva causa que puede sumarse a las cinco anteriores para explicar la escasez eléctrica de la posguerra y que hasta ahora ha recibido menor atención: en 1934-36, las compañías eléctricas firmaron un pacto que cartelizó la oferta y reconoció a la empresa Saltos del Duero como productor dominante a cambio de que renunciara a distribuir electricidad en algunos de los mercados abastecidos por el resto de las sociedades. Como consecuencia de esta división de papeles se produjo un enfrentamiento de intereses: a las empresas encargadas de la distribución les convenía el máximo incremento de producción posible mientras que a Saltos del Duero le preocupaba ante todo garantizar la salida de su producción. Y Duero erró en sus cálculos de previsión de consumo. Al defender su monopolio en la producción y al mismo tiempo no conceder fiabilidad a las peticiones de las empresas distribuidoras para que aumentara su capacidad productiva, entorpeció la respuesta de la oferta a las crecientes necesidades de la demanda 8 .

Las explicaciones basadas fundamentalmente en la falta de incentivos provocada por la congelación de las tarifas eléctricas ordenada por el Estado, pueden verse en Sudrià (1994), p. 334; (2007), p. 45; Catalán (1994), pp. 388-9; (1995), pp. 257-264; Gómez Mendoza (2000), p. 84; (2007), pp. 459 y 470; Barciela, López, Melgarejo y Miranda (2001), pp. 143-144, y Pueyo (2007), pp. 115-122. Este motivo ya fue indicado en su tiempo por el ingeniero del INI Ignacio Sirvent. Gómez Mendoza (2000), p. 75. Gómez Mendoza (2000), pp. 71-72.

Sudrià (2001), p. 16.

Conviene aclarar que llamamos distribuidoras a las empresas pertenecientes al grupo hidroeléctrico para diferenciarlas de Saltos del Duero, tal y como se definen en ocasiones a sí mismas en el acuerdo de 193436, pero tanto aquéllas como Duero eran a la vez productoras y distribuidoras y no se limitaban a un solo aspecto del negocio eléctrico. 
La construcción de nuevas centrales también fue frenada por la escasez de materias primas y de divisas que marcó aquellos años ${ }^{9}$.

En la mentalidad de los gestores de Duero pesó, sin duda, el recuerdo de las consideraciones que se habían vertido en numerosas ocasiones durante el decenio de 1920 sobre la locura que suponía su proyecto de aprovechamiento del río Esla, así como las dificultades para asegurar la colocación de su producción en los años treinta. De este modo, la falta de previsión de Saltos del Duero y la peculiar organización del sector eléctrico español, que enfrentaba los intereses expansionistas de los distribuidores con los más cautelosos del principal productor, pueden unirse a las razones más frecuentemente citadas hasta el momento para explicar por qué la iniciativa privada no llegó a tiempo de evitar las restricciones energéticas que padeció España durante el primer franquismo.

Dedicamos las páginas siguientes a estudiar esta nueva causa. Para ello, los dos próximos apartados analizan el reparto de papeles entre las empresas del grupo hidroeléctrico y Saltos del Duero. Repasamos, por un lado, los antecedentes y las causas del pacto, y por otro lo describimos con cierto detalle ${ }^{10}$. El apartado siguiente expone lo sucedido durante la Guerra Civil: los éxitos económicos que alcanzó Saltos del Duero frente a las penurias que pasaron sus competidoras Hidroeléctrica Española e Hidroeléctrica Ibérica. El último apartado analiza en profundidad la nueva causa de la restricción eléctrica que proponemos -contraponiéndola a la de las bajas tarifas-, y recoge nuestras conclusiones.

\section{Los antecedentes del convenio de 1934-36}

La necesidad de pactos entre las empresas eléctricas en el decenio de 1930 surgió de la tardía introducción del Banco de Bilbao en el negocio eléctrico, donde ya estaban asentados desde hacía tiempo el Banco de Vizcaya y el Banco Urquijo. El Vizcaya había configurado un grupo eléctrico cuyos vértices eran Hidroeléctrica Ibérica, Hidroeléctrica Española y Electra del Viesgo ${ }^{11}$. El Urquijo era propietario de Unión

9 Gómez Mendoza (2007), vol I, p. 443.

10 Empleamos una concepción de estructura industrial restringida a las relaciones entre empresas que determinan la posición de cada una en el sector, dejando fuera de nuestra explicación otros aspectos que también le pertenecen, como el control del mercado, el grado de integración vertical, las barreras de entrada o la diferenciación del producto. Para un resumen de la teoría básica de la organización industrial puede consultarse Carlsson (1981), pp. 126-7.

11 Luego amplió el grupo con participaciones en Mengemor, la Compañía Sevillana de Electricidad y Electra de Lima. De este modo, a la altura de 1930, un elevado porcentaje de la producción y distribución eléctri- 
Eléctrica Madrileña y sus filiales. El nuevo contendiente al que hubo que admitir en el compartimentado y monopolizado mercado eléctrico nacional llegaba con el arma poderosa de la gran producción de su central del Esla, inaugurada en 1935, y con la capacidad financiera suficiente como para atreverse a competir en la distribución. Veámoslo desde el principio.

Las relaciones entre Saltos del Duero y las empresas agrupadas en lo que se conoció como grupo hidroeléctrico, que aunaba a las del Vizcaya y el Urquijo, arrancaron bajo el signo de la confrontación. En cuanto la nueva compañía se constituyó en 1918, los responsables de Hidroeléctrica Ibérica, Hidroeléctrica Española y el Banco de Vizcaya se lanzaron a la creación de una sociedad cuyo único objetivo se resumía en entorpecer la marcha de la empresa del Banco de Bilbao, poniendo en cuestión sus concesiones sobre el río Duero e impulsando una fuerte campaña política y propagandística contraria a sus intereses en círculos de poder y de opinión españoles y portugueses ${ }^{12}$. La Sociedad de Electrificación Industrial (SEI), que así se llamó el invento, rescató unos supuestos derechos de concesiones de saltos de la casa Vickers cedidos oportuna y gratuitamente a su filial, la Sociedad Española de Construcción Naval, que formaba parte de la SEI junto con Altos Hornos de Vizcaya. Aunque la base legal de tales derechos no era nada clara, consiguieron sembrar la duda en las autoridades del país vecino, que debían dar su consentimiento para el aprovechamiento de la parte internacional del río, y, lo que es más grave, también en el seno del consejo de administración del Banco de Bilbao. La paralización duró ocho años, hasta que, en 1926, los esfuerzos del ingeniero jefe de Saltos del Duero, José Orbegozo, y de algunos consejeros de la sociedad, como Horacio Echevarrieta y Domingo Epalza, dieron sus frutos al ratificarse definitivamente las concesiones y firmarse un acuerdo con Portugal ${ }^{13}$. En toda la estrategia del grupo del Banco de Vizcaya desempeñó un destacado papel protagonista Fernando María de Ybarra y de la Revilla, marqués de Arriluce, presidente de Hidroeléctrica Ibérica y de Hidroeléctrica Española, vicepresidente de la Sociedad Española de Construcción Naval y de Altos Hornos de Vizcaya y consejero del banco, que ostentó a su vez la presidencia de la SEI y que dirigió la labor de oposición a Duero ${ }^{14}$.

El objetivo de Arriluce era doble. Por un lado, pretendía retrasar el mayor tiempo posible la entrada de Saltos del Duero en el sector, algo que se logró con creces; pero, por otro lado, había que avanzar de forma ineludible hacia algún tipo de acuerdo con

\footnotetext{
ca española estaba en manos de un trust articulado en torno a la entidad de crédito vizcaína. Sólo Cataluña quedaba fuera por completo de los intereses eléctricos del Banco de Vizcaya. San Román (2006a), p. 28. Díaz Morlán (1998); Tedde y Aubanell (2006), p. 261; Valdaliso (2006), pp. 113-114.

Díaz Morlán (1998).

Sobre el marqués de Arriluce pueden consultarse Rojo (2000) y Díaz Morlán (2002).
} 
Duero fortaleciendo la posición propia. En este segundo objetivo las cosas no resultaron tan fáciles para las sociedades presididas por Arriluce, ya que Saltos del Duero se negó a negociar con Hidroeléctrica Ibérica si antes no se aceptaban sus derechos sobre el río. Sin embargo, el empeoramiento del mercado eléctrico en el decenio de 1930 acercó las posturas de los contendientes. La inminente llegada de la abundante y barata energía de la central del Esla se sumó al incremento de la oferta en el decenio anterior. Tales circunstancias imponían un acuerdo, porque en caso contrario Duero incurriría en costosas inversiones y el grupo hidroeléctrico sufriría las negativas consecuencias de una competencia en precios.

Pedro Tedde y Anna Aubanell han explicado con detalle el proceso negociador entre Saltos del Duero y el grupo hidroeléctrico a partir de $1926^{15}$. Arriluce y sus compañeros se vieron obligados a aceptar la legalidad de las concesiones de Duero y a buscar algún tipo de colaboración, también pretendida por los contrarios. Los primeros intentos negociadores, en 1927 y 1929, fracasaron porque no existía dentro de Duero una estrategia común entre los socios nacionales y los extranjeros, que deseaban dominar el negocio de la distribución. Por fin, la victoria interna de los socios nacionales hizo posible el inicio de nuevas conversaciones con el grupo hidroeléctrico en noviembre de 1930. Al tiempo que alcanzaban un primer acuerdo con Unión Eléctrica Madrileña, Orbegozo y Epalza propusieron la creación conjunta de una gran distribuidora nacional. Esta idea fue rechazada en junio de 1931 tras varias reuniones, posiblemente porque los representantes del grupo hidroeléctrico todavía creían que podían mantener una ventaja determinante sobre el nuevo competidor ${ }^{16}$.

Las importantes consecuencias de la entrada de Saltos del Duero en el mercado eléctrico español se ponen de manifiesto si se considera la capacidad de la central del Esla: ella sola podría ofrecer hasta 400 millones de $\mathrm{kWh}$ al año, casi dos tercios de la energía eléctrica que podían producir Hidroeléctrica Ibérica, Electra del Viesgo e Hidroeléctrica Española en conjunto. La estrategia del consejo de Duero se centró en realizar una demostración de fuerza mientras se preparaban para la terminación de la central, trazando las líneas de alta tensión hasta Madrid y el País Vasco y mostrando su determinación de participar en el negocio de la distribución, en franca competencia con las empresas ya establecidas. Tal estrategia, expuesta por Orbegozo ante el consejo, se concretó en el País Vasco en un acuerdo firmado con Cooperativa Eléctrica de Bilbao y en un estudio para crear una distribuidora en Guipúzcoa.

\footnotetext{
15 Tedde y Aubanell (2006), pp. 261-266; véase también Valdaliso (2006), pp. 110-112.

16 En el propio consejo de administración de Saltos del Duero se temían los problemas financieros que acarrearía la entrada directa en la distribución y no pocos consejeros abogaban por llegar a acuerdos con las empresas existentes. Archivo de Iberdrola, Actas del Consejo de Administración de Saltos del Duero (en adelante, CASD), acta del 28/09/1931.
} 
En Madrid, Duero mostró su determinación al presentarse al concurso para la electrificación de la Ciudad Universitaria ${ }^{17}$.

La "demostración estratégica" diseñada por Orbegozo dio finalmente sus frutos, pues en enero de 1933 se reanudaron las negociaciones. En noviembre entró en ellas la Unión Eléctrica Madrileña del Banco Urquijo y, una vez acordado dentro del grupo hidroeléctrico el "reparto de sacrificios" que iba a suponer el acuerdo con Duero, se aprobaron en julio de 1934 las bases iniciales del convenio, que entró en vigor un año después y fue elevado a escritura pública el 1 de febrero de 1936. El acuerdo significó, en definitiva, el triunfo de la idea que un decenio atrás había lanzado el consejero de Saltos del Duero, Julio Arteche, a sus compañeros de consejo. Ante el tamaño del reto empresarial que suponía el aprovechamiento del río Duero, no era posible "encontrar aportadores nacionales sin rozar el problema de la competencia", y por ello se hacía necesario el entendimiento entre los escasos protagonistas del capitalismo español ${ }^{18}$.

\section{Las cláusulas del pacto}

Tres eran las directrices esenciales que contenía el pacto firmado ante notario a principios de 1936: en primer lugar, se reconocía a Saltos del Duero el papel de "productor ilimitado" de energía eléctrica una vez que las empresas terminaran de construir las concesiones de las que disponían. En segundo lugar, se creaba un "grupo hidroeléctrico" que aunaba a las sociedades Unión Eléctrica Madrileña, Electra de Castilla, Saltos del Alberche, Hidroeléctrica Española, Electra de Viesgo e Hidroeléctrica Ibérica y les otorgaba el papel de "distribuidoras exclusivas" de la energía de Duero en muchos de sus mercados tradicionales, que pasaban a ser mercados compartidos con Saltos del Duero. La tercera directriz, de carácter más general, recogía el esfuerzo conjunto de todos los firmantes para intensificar el consumo de energía eléctrica, especialmente el doméstico ${ }^{19}$.

Archivo de Iberdrola, CASD, acta del 14/12/1931; y Actas del Comité de Gerencia (en adelante, CGSD), actas de 29/04/1930, 20/12/1930, 16/01/1931 y 30/11/1931.

18 Archivo de Iberdrola, CASD, acta del 22/09/1926. En este sentido, resulta significativo el cálculo realizado por Isabel Bartolomé del peso de los recursos básicos (capital desembolsado más obligaciones en circulación) del sector eléctrico dentro del conjunto de las sociedades anónimas españolas: de un total de 21.000 millones de pesetas a la altura de 1935, 4.300, una quinta parte, correspondían a las empresas eléctricas. Bartolomé (2007), p. 89.

Archivo de Iberdrola, Salto de Ricobayo, "Contrato de suministro de energía eléctrica y de prestación de servicios que celebran las sociedades Saltos del Duero, Unión Eléctrica Madrileña, Eléctrica de Castilla, Saltos del Alberche, Hidroeléctrica Española, Electra del Viesgo, Cooperativa Electra Madrid, Hidroeléctrica Ibérica, Cooperativa Eléctrica de Langreo y Energía e Industrias Aragonesas". En adelante lo citaremos como "Contrato 1936". 
El acuerdo dividía el mercado eléctrico español en tres categorías: los mercados exclusivos, los mercados compartidos y los libres. En los mercados exclusivos se reconocía el monopolio de producción y de distribución a las empresas que allí operaban. Eran, en definitiva, los territorios del grupo hidroeléctrico en los que Saltos del Duero no podía entrar de ninguna forma y, por el mismo principio, la zona de Duero vetada a las empresas del grupo hidroeléctrico. Sólo tres de las sociedades firmantes del acuerdo tenían capacidad de poseer mercados exclusivos puesto que eran autosuficientes para abastecerlos: Saltos del Duero, Hidroeléctrica Española e Hidroeléctrica Ibérica. Los mercados compartidos eran zonas explotadas por empresas del grupo hidroeléctrico en las que éstas aceptaban actuar como distribuidoras exclusivas de la producción eléctrica de Duero. Para ello se les obligaba a la compra de un cupo de energía de la empresa del Banco de Bilbao y se fijaban unos precios mínimos para esta energía. Las dos zonas compartidas eran la norte/noroeste y la zona centro, en referencia a su ámbito geográfico. La zona norte/noroeste comprendía los mercados tradicionales de Electra del Viesgo y los no exclusivos de Hidroeléctrica Ibérica, y la zona centro agrupaba los no exclusivos de Hidroeléctrica Española así como los mercados de Saltos del Alberche y Eléctrica de Castilla, tal y como queda reflejado en el cuadro 1.

Cuando las empresas del grupo hidroeléctrico de la zona norte/noroeste hubieran consumido toda la energía del cupo y se hiciera necesario producir más, Saltos del Duero tendría derecho a participar como productor en la mitad del aumento de consumo de la Ibérica y de Viesgo. Una vez agotadas las capacidades de ambas empresas, Duero suministraría todos los aumentos de consumo salvo en Guipúzcoa, Álava y Navarra, donde se reservaría la mitad de la producción de esos aumentos a Energía e Industrias Aragonesas. A cambio, esta empresa se comprometía a no colocar su fuerza hasta que llegara ese momento en la zona compartida por Duero y el grupo hidroeléctrico ${ }^{20}$.

Por último, el pacto de 1936 reconocía la existencia de los llamados mercados libres, constituidos por todas las provincias y partes del territorio nacional que no se mencionaban expresamente en el acuerdo. Haciendo honor a su nombre podían ser abastecidos por cualquier empresa puesto que, en definitiva, quedaban al margen del convenio. Había, además, algunos casos especiales que se mencionaban en el acuerdo y que, por tanto, no formaban parte del mercado libre ${ }^{21}$.

También en Asturias, cuando se agotara la capacidad de la Ibérica y de Viesgo, se reservaba una parte del derecho de producción a Cooperativa Eléctrica de Langreo, que se obligaba a no aumentar su capacidad. A este grupo pertenecían Ávila y Segovia, en donde existían contratos entre algunas empresas del grupo hidroeléctrico y de Saltos del Duero y que se decidió dejarlas momentáneamente fuera del acuerdo hasta estudiar el asunto y alcanzar un consenso sobre ellas. También era especial la propia capital burgalesa y un radio de 40 kilómetros a su alrededor, que quedaba excluida de la zona norte/oeste y sería atendida por 
CUADRO 1

MERCADOS EXCLUSIVOS Y COMPARTIDOS SEGÚN EL PACTO DE 1936

\begin{tabular}{|c|c|c|c|}
\hline & Saltos del Duero & Hidroeléctrica Ibérica & Hidroeléctrica Española \\
\hline \multirow[t]{2}{*}{$\begin{array}{l}\text { Mercados } \\
\text { exclusivos }\end{array}$} & $\begin{array}{l}\text { Valladolid, Cáceres, } \\
\text { Badajoz, Zamora, } \\
\text { Salamanca, Soria, León*, } \\
\text { Palencia*, Burgos* }^{*}\end{array}$ & Huesca, Zaragoza & $\begin{array}{l}\text { Teruel, Castellón, Valencia, } \\
\text { Alicante, Murcia, Almería, } \\
\text { Cuenca y Albacete }\end{array}$ \\
\hline & Electra del Viesgo & Hidroelé & $\begin{array}{c}\text { H. Española } \\
\text { Unión Eléctrica Madrileña } \\
\text { Saltos del Alberche } \\
\text { Eléctrica de Castilla }\end{array}$ \\
\hline $\begin{array}{l}\text { Mercados } \\
\text { compartidos } \\
\text { con Saltos } \\
\text { del Duero }\end{array}$ & $\begin{array}{l}\text { Zona norte- noroeste: } \\
\text { Coruña, Lugo, Orense, } \\
\text { Pontevedra, Oviedo, } \\
\text { Santander, León**, } \\
\text { Palencia**, Burgos** }^{* *}\end{array}$ & $\begin{array}{l}\text { Zona norte-noroeste } \\
\text { País Vasco, Navarra, } \\
\text { Logroño, Burgos }{ }^{\star \star \star}\end{array}$ & $\begin{array}{c}\text { Zona centro: } \\
\text { Madrid, Toledo, Guadalajara } \\
\text { y Ciudad Real }\end{array}$ \\
\hline \multicolumn{4}{|c|}{$\begin{array}{l}\text { Sólo les correspondían con carácter exclusivo las zonas de estas provincias no asignadas al grupo hidroeléctrico } \\
\text { como mercados compartidos o zonas de influencia. } \\
\text { La Vecilla y Liaño (León), Cervera de Pisuerga, Saldaña, Carrión de los Condes y Astudillo (Palencia), y Castrojeriz } \\
\text { y Villadiego (Burgos). }\end{array}$} \\
\hline
\end{tabular}

Fuente: Archivo de Iberdrola, Salto de Ricobayo, Contrato 1936, pp. 27-29.

Las directrices del pacto enumeradas al inicio de este epígrafe impedían, como consecuencia del reparto de papeles, que las empresas del grupo hidroeléctrico emprendieran nuevas construcciones. Tampoco podían utilizar nuevos medios de producción, ni emplear energía de terceros. Estas prohibiciones contemplaban algunas salvedades. Por ejemplo, el principio de no construcción no se aplicaba a los lugares que quedaban fuera del convenio, siempre que la energía obtenida en ellos se colocara fuera de las zonas compartidas con Saltos del Duero. En la misma línea, se autorizaba a Electra del Viesgo y a Hidroeléctrica Ibérica a terminar las instalaciones que tenían en marcha y algunos proyectos ya aprobados, previa garantía de que la producción obtenida con las mejoras no excediera el 10 por ciento del total que

empresas menores con las que Electra del Viesgo y la Ibérica tenían contratos de suministro de energía y exclusiva de distribución. Sin embargo, Viesgo y la Ibérica se comprometían a procurar que esas pequeñas distribuidoras no extendieran sus líneas hacia la zona de Saltos del Duero. 
alcanzasen antes de llevarlas a cabo ${ }^{22}$. A Hidroeléctrica Española, por su parte, se le permitía ampliar cuanto quisiera su capacidad de construcción en sus mercados exclusivos siempre que la energía obtenida no saliera de ellos, ni desplazara energía de terceros a los mercados compartidos con Saltos del Duero ${ }^{23}$.

El convenio de 1936 reforzaba el reparto del mercado y el buen entendimiento entre las sociedades firmantes mediante la mutua posibilidad de participar en las ampliaciones de capital. Sin presentarlo como una obligación, Saltos del Duero podía interesarse, hasta un límite del 25 por ciento, en las ampliaciones de capital de las sociedades del grupo y por reciprocidad éstas podían repartirse entre ellas, si lo deseaban, hasta un 25 por ciento de las ampliaciones de Duero. Sólo Hidroeléctrica Española no participaba de esta posibilidad para evitar la entrada de Duero en su capital. El buen entendimiento entre las sociedades quedaba además asegurado con la creación de un comité mixto con dos delegaciones, una por cada zona compartida, para interpretar las cláusulas del convenio y mediar en caso de conflicto.

Es difícil exagerar la importancia que los firmantes otorgaron al convenio de 1936. Bastan dos últimos apuntes al respecto para ilustrarlo: por un lado se conminaba a las sociedades que participaban en él a desarrollar una labor conjunta para que todas las empresas que operaban en los mercados compartidos fueran sumándose al acuerdo. Por otro lado, y como colofón, se establecía un "cumplimiento coactivo" del convenio, de tal modo que si una sociedad lo infringía las demás renunciaban a disolver el acuerdo con ella. Antes bien, a la infractora se le obligaría a volver al cumplimiento del pacto, previo pago de la indemnización de los daños y perjuicios causados. Esta firmeza sólo se explica porque, en palabras textuales de los firmantes, las sociedades contratantes consideraban "(...) fundamental que el convenio se mantenga vivo y eficaz". En definitiva, su existencia aseguraba el reparto del mercado y la desaparición de la competencia. Todos los firmantes habían cedido terreno ante la amenaza de Saltos del Duero, pero el acuerdo había creado un cártel que, al menos, aseguraba su supervivencia.

\footnotetext{
22 En concreto se trataba del segundo salto de Navia y de construcciones relacionadas con la central de Lafortunada: un embalse en el Cinca, un salto en el canal de Barrosa-Chisagüés al Cinca, y una prolongación del salto auxiliar de las obras del Cinca.

23 Por su parte, a Saltos del Alberche se le permitiría terminar sus obras en el río del mismo nombre si el Estado le obligaba, para no perder la concesión. En cuanto a la prohibición relativa a emplear energía de terceros, se dejó al margen la que el grupo estaba adquiriendo antes de la firma del acuerdo, la que se había comprometido a comprar por convenios previos y, muy especialmente, la procedente de los canales de Lozoya, cuya producción convenía absorber para no perturbar el mercado.
} 


\section{Las consecuencias de la Guerra Civil en las principales empresas eléctricas}

La Guerra Civil española afectó de modo muy diferente a las principales empresas eléctricas españolas y dejó en suspenso el pacto firmado a principios de 1936. Saltos del Duero mantuvo sus activos y su capacidad decisoria en la España de Franco durante todo el conflicto. Hidroeléctrica Española quedó dividida: sus activos permanecieron en la España leal a la República y su consejo de administración, así como la mayoría de sus directivos, quedaron o se incorporaron a la España de Franco. Hidroeléctrica Ibérica vivió la situación más compleja: una parte de los activos de la sociedad quedó en terreno nacional y funcionó bajo la dirección de la Distribuidora Guipuzcoana, pero la central térmica de Burceña, en Vizcaya, y, sobre todo, la central hidroeléctrica de Lafortunada, en Huesca, estuvieron buena parte de la guerra bajo control republicano, aunque la energía de esta última no pudo ser aprovechada por la industria vasca, su cliente habitual hasta el estallido de la contienda. Al mismo tiempo y hasta la caída de Bilbao, en el verano de 1937, continuó actuando un consejo paralelo de la Ibérica en la capital vizcaína, con la presencia del Gobierno de Euzkadi, lo que también le ocurrió a Duero, pero en el caso de este último sin auténtica capacidad para decidir sobre unos activos que quedaron por completo fuera de su control.

Tal disparidad de situaciones provocó consecuencias también muy diversas. Desde el exclusivo punto de vista del negocio, la guerra fue para Saltos del Duero una ocasión única de completar su hegemonía en su mercado natural mientras que Hidroeléctrica Española e Hidroeléctrica Ibérica se conformaron con sobrevivir. Ambas empresas sufrieron la desaparición de notables miembros de sus consejos de administración, como su presidente común, el marqués de Arriluce, asesinado en septiembre de 1936. El consejo de administración de Hidroeléctrica Española pasó la guerra sin tener apenas noticias sobre la situación de sus instalaciones. La inestabilidad de la España republicana se hizo sentir de forma creciente a lo largo del conflicto, especialmente en la empresa madrileña.

\subsection{El éxito de Saltos del Duero}

Al iniciarse la contienda, los militares sublevados se aprestaron a cortar de inmediato los envíos de fuerza de la central del Esla a Bilbao, e hicieron otro tanto con los que la Ibérica llevaba desde su central de Lafortunada en el río Cinca ${ }^{24}$. Durante casi 
un año, Vizcaya sufrió como consecuencia una gran escasez de energía eléctrica que vino a sumarse a la falta de carbón y que dificultó sobremanera la marcha de su industria, hasta que la toma de la ciudad por las tropas nacionales en junio de 1937 logró reunificar el centro productivo de Zamora con el centro consumidor de Bilbao. El consejo de administración de Saltos del Duero, rehecho de nuevo, se reunió con prontitud en la capital vizcaína para dar celeridad al cumplimiento de las instrucciones de las autoridades franquistas, y la central del Esla constituyó desde ese momento el origen fundamental de la energía que necesitaba la industria pesada vasca, convertida en industria armamentística de esencial importancia para las posibilidades de victoria de los alzados. Por su parte, la central de Hidroeléctrica Ibérica en el Cinca se mantuvo en zona republicana hasta junio de 1938 y después necesitó varios meses para su reparación. Mientras tanto, se exigió a Duero que trazara el enlace Seira - Lafortunada, que serviría para garantizar el abastecimiento a Bilbao desde las centrales catalanas en caso de que fallase el suministro procedente del Esla, como así ocurrió durante los últimos meses de la guerra ${ }^{25}$. La Ibérica, incapacitada para suministrar la fuerza de sus centrales, vio así cómo Duero se fortalecía al albur de la lógica bélica y de las necesidades del ejército nacional ${ }^{26}$.

Otra de las consecuencias positivas que tuvo para Duero la Guerra Civil fue el impulso que dieron las autoridades franquistas a la conclusión de lo que la empresa consideraba como su mercado propio, que abarcaba Castilla, León y Extremadura. Estas regiones se hallaban, casi en su totalidad, en zona nacional desde el principio de la sublevación o pasarían a estarlo durante los primeros meses de la contienda, y el interés del gobierno de Burgos estribaba en suministrar la fuerza que necesitaban las minas leonesas y las ciudades bajo su dominio. Para lograrlo se apoyó en la empresa más fuerte de las existentes, que era, con gran diferencia, Saltos del Duero, forzando las negociaciones con las diversas sociedades distribuidoras de cada provincia o localidad que aún no habían caído en la esfera de influencia de la compañía bilbaína. Una tras otra, las pequeñas eléctricas se vieron obligadas a firmar contratos de compra de fuerza a largo plazo o a ceder su negocio a cambio de una compensación económica, y, al acabar la contienda, Duero contaba con una decena de filiales y el control casi absoluto de su mercado propio ${ }^{27}$.

\footnotetext{
25 "Ninguna sociedad se halla tan preparada como lo estamos nosotros para realizar con la necesaria diligencia ese trabajo". Archivo de Iberdrola, Actas de la comisión delegada de Saltos del Duero (en adelante, CDSD), reunión del 28 de julio de 1938. Archivo de Iberdrola, Actas del Consejo de Administración de Hidroeléctrica Ibérica, reunión del 11 de febrero de 1938 (facilitada por Jesús Ma Valdaliso), y CASD, acta de 14/12/1938.

27 Durante la guerra la expansión de Duero tuvo lugar sobre todo en las provincias de Burgos, Soria, León y Zamora, que eran aquéllas en las que más dificultades había encontrado en los años prebélicos. Sólo quedó fuera de su dominio El Porvenir de Zamora, pero por poco tiempo, porque fue adquirida por Iberduero en 1947. Véase Díaz Morlán (2006), pp. 294-302.
} 
Gracias a Duero, el nuevo Estado franquista pudo poner a trabajar a todo ritmo a la industria pesada del norte, abandonada intacta por el gobierno nacionalista vasco de Aguirre. Con este servicio, en 1938 llegaron los beneficios, pues los márgenes de explotación de Duero, al igual que los de toda empresa hidroeléctrica, eran excelentes una vez reducidos los costes financieros ligados a las construcciones ${ }^{28}$. En 1940, veintidós años después de su fundación, Saltos del Duero pudo por fin repartir un dividendo a sus accionistas del 3,5\% sobre el capital, que se elevaría al 4,5\% en 1941 y al 6\% en 1942.

En resumen, y a pesar de los problemas que generó el aliviadero de la presa del Esla, la situación de Saltos del Duero al terminar la contienda era halagüeña ${ }^{29}$. La destrucción de capital fijo había sido mínima, la absorción del mercado propio casi había concluido y el pacto alcanzado en 1936 con el grupo hidroeléctrico le prometía la exclusividad de nuevas construcciones y la colocación de la fuerza producida por su central del Esla. La colaboración estrecha con las nuevas autoridades le garantizaba a su vez el favor del Estado, y la debilidad arrostrada por Hidroeléctrica Ibérica durante los años de guerra le auguraba una posición de fuerza frente a quien había sido su principal contendiente en la lucha por los derechos del río castellano y la distribución de la energía en el País Vasco. A la altura de 1939-40, Saltos del Duero emergía por fin como una empresa hidroeléctrica consolidada que podía repartir dividendos a sus accionistas y plantearse nuevos proyectos para expandir su producción.

\subsection{El fracaso de Hidroeléctrica Ibérica}

Comparada con la de Saltos del Duero, la historia de Hidroeléctrica Ibérica durante la Guerra Civil representa la otra cara de la moneda. Fue, con mucho, la empresa que más sufrió los avatares de la contienda. Nada más iniciarse la guerra, su presidente, Fernando Ybarra Revilla, marqués de Arriluce, a quien José Calvo Sotelo había encomendado la responsabilidad de buscar financiación para el alzamiento, fue detenido y llevado al barco-prisión Cabo Quilates, anclado en la ría de Bilbao, en donde fue asesinado en septiembre de $1936^{30}$. El resto de los miembros del consejo de administración sufrieron suerte diversa, pero pronto pudo reagruparse en San Sebastián tras la toma de la ciudad vasca por las tropas nacionales. Su capacidad de decisión sobre los activos de la compañía quedó limitada a los que poseía en Guipúzcoa, ya que la

\footnotetext{
28 Pueyo (2007), p. 250.

29 Sobre los problemas que generó el aliviadero de la presa véase Díaz Morlán (2006), pp. 291-294.

$30 \quad$ Estos acontecimientos han sido narrados en Díaz Morlán (2002), p. 262.
} 
central térmica vizcaína de Burceña y la oscense de Lafortunada siguieron bajo control republicano, la primera hasta la caída de Bilbao en junio de 1937, y la segunda hasta el fin de la bolsa de Bielsa, en junio de 1938.

En estas condiciones, no es de extrañar que los resultados obtenidos por Hidroeléctrica Ibérica durante la guerra fueran negativos. La producción cayó de 302.000 kWh en 1935 a un mínimo de 177.500 en 1937, alzándose hasta 347.000 en 1939, y se suspendió el reparto de dividendo hasta $1938^{31}$. No son datos que indiquen una catástrofe para la Ibérica, desde luego, pero sí la alejan del comportamiento exitoso de Saltos del Duero, que salió reforzada de la contienda y aprovechó la debilidad de su contrincante para mejorar su posición relativa en el sector. Cuando terminó la contienda, Duero pudo alegar diversos méritos ante las nuevas autoridades, incluidos el suministro a la industria vasca en sustitución de la Ibérica, el enlace SeiraLafortunada y la satisfacción de la demanda eléctrica de las provincias castellanas y las minas leonesas. Toda una extensa hoja de servicios frente a su rival.

\subsection{Hidroeléctrica Española: una empresa sin empresarios ${ }^{32}$}

Desde agosto de 1936, el consejo de administración de Hidroeléctrica Española estuvo intervenido por las autoridades del gobierno de la República en cumplimiento de la legislación promulgada a tal efecto ${ }^{33}$. Quedó tan sólo uno de los antiguos consejeros, de adscripción política republicana, y se sumaron los tres representantes del gobierno prescritos por las nuevas leyes, así como un representante del personal técnico administrativo y otro del personal obrero. Se formó, además, un comité de control, integrado por trabajadores, cuyas funciones debían limitarse a la inspección de la actividad industrial y a la presentación de sugerencias al consejo. De las dos tareas asignadas, el comité de Hidroeléctrica Española se dedicó con especial afán a la segunda y, en realidad, se convirtió en una activa oficina puente entre los trabajadores y el consejo, diseñada para hacer llegar todo tipo de peticiones y quejas.

La coexistencia de propietarios, interventores del gobierno central y comité obrero hizo del consejo de administración de Hidroeléctrica Española un órgano

\footnotetext{
$31 \quad$ Antolín (2006), pp. 175 y 186.

32 Véase San Román (2006a), pp. 81-82; (2006b), pp. 325-346. Para la redacción de este epígrafe se han utilizado como fuente principal las actas del consejo de administración de la empresa correspondientes a los años bélicos, conservadas en el Archivo de Hidroeléctrica Española. 
muy politizado y poco operativo, saturado por cuestiones escasamente relacionadas con la electricidad. Este peculiar consejo de administración, sin profesionales de la gestión y con la supervivencia de la empresa como único plan estratégico, supo, sin embargo, protegerse de los desórdenes revolucionarios que llevaron a la ruina a muchas otras entidades industriales en la España de la República, y supo también preservar su estructura jurídica de sociedad anónima.

Hidroeléctrica Española mantuvo durante la guerra una cierta normalidad en dos aspectos cruciales: su ritmo de producción y su mercado, que funcionó a pesar de las distorsiones. La práctica inexistencia de destrucciones en el capital físico de la sociedad explica el mantenimiento de la producción en niveles no muy lejanos a los de 1935, con un año incluso por encima del nivel de preguerra ${ }^{34}$. El funcionamiento del mercado constituye un hecho más llamativo si se tiene en cuenta el grado de descomposición que alcanzó la España de la República. El pacto de 1936 fue suspendido en el mes de julio, por razones obvias, y se paralizaron todos los planes de desarrollo del mercado que tenía previstos la empresa. Sin embargo, Hidroeléctrica Española siguió alentando durante la guerra los acuerdos de entendimiento entre empresas eléctricas que permitieran repartir clientes y evitar, en lo posible, el descenso de los precios.

Los dos problemas principales que obstaculizaron el funcionamiento del mercado en aquellos tiempos de conflicto fueron el fraude eléctrico y los impagados, que explican el descenso de los ingresos de la sociedad durante la guerra. Por su parte, los gastos llevados a la cuenta de pérdidas y ganancias aumentaron considerablemente en los años 1938 y 1939 debido a la decisión de la dirección de la empresa de introducir correcciones por aumento de gastos de explotación y amortizar en varios ejercicios los quebrantos ocasionados por la guerra. El descenso de los ingresos, la inflación y el aumento de los gastos redujeron el margen de beneficios, y las correcciones contables hechas en la inmediata posguerra explican, en parte, la drástica disminución de los dos últimos ejercicios. Así, de casi 20 millones de pesetas de beneficios obtenidos en 1935, se pasó a poco más de 10 en 1939, debilitándose, aunque no gravemente, la posición financiera de la empresa.

En definitiva, a pesar del regular funcionamiento que mantuvo la empresa y de la escasa destrucción de capital físico que hubo de afrontar, Hidroeléctrica Española perdió posiciones respecto a su principal rival entonces, Saltos del Duero, cuya situación geográfica le convirtió, como ya se ha visto, en pieza central para el aprovisionamiento de la retaguardia franquista. 34 La producción anual osciló entre los 400 millones de kwh de 1936 y los 550 de 1938, frente a los 464 millo-
nes de kwh obtenidos en 1935. Memorias de la empresa, años de 1935-1939. 


\section{Una nueva perspectiva para el debate sobre restricción eléctrica}

El establecimiento de bajas tarifas por parte del nuevo régimen salido de la Guerra Civil se ha esgrimido como elemento central para explicar las restricciones eléctricas sufridas en la posguerra. La inflación de los años bélicos y postbélicos y la escasez y carestía de combustibles fósiles se sumaron a la decisión política de no incrementar en igual cuantía los precios nominales de la electricidad, lo que provocó la caída de sus precios reales ${ }^{35}$. En el mercado eléctrico español, esta baratura relativa habría impulsado la demanda y deprimido la oferta, pues las empresas del sector, a la vista de las tarifas fijadas por las autoridades, no habrían considerado conveniente para sus intereses realizar las inversiones necesarias para elevar la producción ${ }^{36}$. Se trata de una explicación respaldada por una lógica económica simple -demanda y oferta distanciadas por culpa de un precio fijado de forma artificial-, y creemos que por ese motivo no ha sido cuestionada en los sucesivos trabajos que han estudiado las restricciones eléctricas. Sin embargo, pensamos que la explicación de lo que sucedió es algo más compleja.

Nuestra propuesta de interpretación emana de la consulta de los archivos empresariales de algunas de las empresas protagonistas del sector eléctrico español. Aceptamos sin discusión que unos precios artificialmente bajos incentivaron la demanda de electricidad en hogares y empresas, y que estas últimas sustituyeron combustibles líquidos y sólidos encarecidos por la situación bélica mundial y la autarquía. Sin embargo, a la vista de la documentación consultada rechazamos que las bajas tarifas fueran la causa principal de la escasez de oferta, ya que las empresas eléctricas afectadas no tomaron sus decisiones en función del precio de la electricidad, sino de otras circunstancias que analizaremos a continuación. Es más, las empresas eléctricas dispusieron de capacidad financiera suficiente, proveniente del ahorro nacional -canalizado tanto a través de los bancos como del mercado de capitales-, para llevar a cabo las inversiones requeridas para elevar la producción, y la razón de no emprender tales inversiones a tiempo no radicó en las tarifas.

¿Por qué fue, entonces, tan ineficiente la respuesta de la oferta a los requerimientos de una demanda en auge? Pensamos que, para entender el problema, resulta imprescindible tener en cuenta el pacto firmado en las fechas previas a la Guerra Civil entre Saltos del Duero y las empresas del grupo hidroeléctrico. Este convenio, unido al incremento imprevisible del consumo, a una sequía especialmente aguda, y

Sudrià (1994), p. 334; Pueyo (2006), pp. 368-9; (2007), pp. 184-6.

Sudrià (1994), p. 334; (2007), p. 45; Catalán (1994), pp. 388-9; (1995), pp. 257-264; Gómez Mendoza (2000), p. 84; (2007), pp. 459 y 470; Barciela, López, Melgarejo y Miranda (2001), p. 143. Un buen resumen puede consultarse en Pueyo (2007), pp. 115-122. 
a la tradicional lentitud de la respuesta de la oferta eléctrica -por su carácter de industria capital-intensiva-, agravada por las circunstancias especiales españolas e internacionales, explica de manera suficiente las restricciones eléctricas sufridas en España a partir de $1944^{37}$. Desarrollaremos nuestro argumento a continuación.

Ya ha quedado dicho que Saltos del Duero salió fortalecida de la contienda, mientras que las empresas del grupo hidroeléctrico vieron su posición debilitada. Pronto las aguas volvieron a su cauce, se restableció el convenio de 1936 y la lbérica pudo disponer de su central del Cinca, lo que provocó una disminución de la producción de Duero, compensada desde febrero de 1940 con la llegada de su energía a Madrid, para comenzar a servir a Unión Eléctrica Madrileña e Hidroeléctrica Española a cuenta de sus cupos. En julio de 1940, quince meses después de acabada la guerra, el grupo hidroeléctrico planteó por primera vez en la reunión del comité mixto del convenio la conveniencia de analizar las disponibilidades de energía de Saltos del Duero, "en vista de los considerables aumentos de consumo que est(aba) experimentando el mercado y de los que se v(enían) anunciando para fecha más o menos próxima", en alusión al recién publicado anuncio de la concesión de una fábrica de nitratos en Valladolid. Sin embargo, para el director general de Duero, Ricardo Rubio, la guerra mundial impedía establecer ni siquiera de forma aproximada la fecha de terminación de dicha fábrica -que requería de maquinaria alemana-, por lo que había que proceder con cautela a la hora de meterse en grandes construcciones que exigirían elevados compromisos financieros. En la siguiente reunión del comité mixto las previsiones de consumo del grupo hidroeléctrico le parecieron "algo exageradas" a Ricardo Rubio. En su opinión, a las empresas distribuidoras les convenía disponer de las mayores reservas de energía, pero no así a Duero, que correría con el riesgo de incurrir en un grave exceso de oferta si no atinaba a acomodar las nuevas construcciones con la verdadera marcha del mercado eléctrico. Ello no obstante, Duero se comprometió a poner los medios materiales y humanos para iniciar el estudio de un nuevo salto, y así se hizo a partir de septiembre de $1940^{38}$.

A comienzos de 1942, los "estímulos" y "excitaciones" del gobierno, su "benevolencia" y las ayudas que estaba dispuesto a ofrecer, así como "las presiones del grupo hidroeléctrico y las necesidades del mercado, que exigen un apresuramiento", convencieron a los responsables de Duero de la necesidad de acelerar la construcción

\footnotetext{
37 La importancia del convenio de 1936 para explicar la falta de respuesta de las empresas eléctricas ya ha sido indicada en Díaz Morlán (2006), y ha quedado recogida recientemente por Javier Pueyo como una explicación alternativa de las restricciones. Pueyo (2007), pp. 121-2 y 424.

38 Archivo de Iberdrola, CDSD, actas de 7/09/1940 y 19/10/1940. Por otra parte, la tranquilidad de los responsables de Duero coincide con la apatía del Estado franquista en materia eléctrica en sus primeros años de andadura, y ratifica la idea de que no se percibió la existencia de un problema eléctrico hasta 1942. Gómez Mendoza (2000), p. 72.
} 
del nuevo salto ${ }^{39}$. Pero, en junio, Electra del Viesgo e Hidroeléctrica Ibérica, manteniéndose aún fieles a las cláusulas del convenio, pidieron a Duero su consentimiento para poder iniciar sus propias construcciones de saltos, ante el temor de que el proyecto de Duero no llegara a tiempo de satisfacer la demanda. Ricardo Rubio obtuvo el apoyo del consejo de administración para negar dicho consentimiento, despreciando los temores de las distribuidoras y atendiendo a la esencia del pacto firmado en 1936:

\begin{abstract}
"No hay fundamento suficiente para creer que el déficit eventual podrá ser cubierto mejor con las construcciones que se proponen que con los del nuevo aprovechamiento que activamente prepara Saltos del Duero con el propósito de llevarlo a cabo en cuanto las circunstancias lo permitan (...) El convenio ... nos da en el concepto de predominantes productores la natural compensación de nuestro compromiso general de no distribuir en extensas y provechosas zonas del territorio nacional y toda merma a esta posición nuestra de productores rompería con grave daño para Duero el equilibrio de derechos y obligaciones recíprocos que es la entraña de nuestro estatuto. La aceptación por Duero de la propuesta de Hidroeléctrica Ibérica y Electra del Viesgo equivaldría a concederles una libertad permanente para desbordar el papel característico de distribuidores que tienen atribuido ${ }^{\prime \prime 40}$.
\end{abstract}

A pesar de las afirmaciones de Rubio, las empresas del grupo hidroeléctrico no cedieron y Electra del Viesgo hizo saber con prontitud que mantenía sus pretensiones constructoras. Duero insistió en que "los temores que en orden al interés público del abastecimiento abriga(ba)n las dos empresas" estaban infundados y que él era "el naturalmente llamado a construir exclusivamente por sí" los nuevos saltos, por lo que la postura de Electra del Viesgo suponía "una rotura fundamental y completa" del convenio y autorizaría a Duero "a proceder con igual libertad" como distribuidor en su zona ${ }^{41}$. Se entablaron negociaciones entre ambas partes bajo el criterio de que cualquier cesión de Duero en su papel de productor debía ser compensado en el negocio de la distribución, y finalmente se llegó a un nuevo pacto el 31 de diciembre de 1942, por el cual Viesgo y la Ibérica adquirían el derecho a construir y Duero era compensado con un $50 \%$ de los aumentos de consumo de la zona servida por la Ibérica. Por su parte, Unión Eléctrica Madrileña e Hidroeléctrica Española

\footnotetext{
39 En mayo de 1942, Ricardo Rubio recibió una citación de la Secretaría Técnica del Ministerio de Industria y Comercio para exponer en una reunión "los planes de la Sociedad en orden a las atenciones del consumo". Archivo de Iberdrola, CDSD y CASD, actas de 14/03/1942 y 25/05/1942. Las "excitaciones" del gobierno coinciden en el tiempo con el convencimiento de Suanzes de la necesidad de intervenir en la industria eléctrica. Gómez Mendoza (2000), p. 72.

40 Archivo de Iberdrola, CASD, acta de 13/06/1942.

41 Archivo de Iberdrola, CDSD, acta de 8/07/1942.
} 
también hicieron saber a Duero su deseo de construir un nuevo salto, pero a cambio de su permiso aceptaban compensarle con un porcentaje en la distribución. Las conversaciones con las empresas distribuidoras de la zona centro llevaron a alcanzar acuerdos similares en marzo y mayo de 1944, aunque limitando a un 33\% la parte de los aumentos de consumo a la que Duero obtenía acceso. En suma, ambos convenios suponían el fin del pacto de 1936 porque terminaban con la división de papeles entre productora y distribuidoras, que pronto se había demostrado contraproducente para la buena gestión de los intereses de todas las partes implicadas ${ }^{42}$.

La decisión de construir el segundo salto de Duero también estuvo marcada por la prudencia ante el recuerdo de los problemas de exceso de oferta de la década de 1930. Se presentaron dos opciones al consejo de administración de Duero. La primera suponía un aumento de la producción anual de 700 millones de kWh y la segunda de 350, y esta última se consideró más que suficiente para cubrir los posibles incrementos de demanda futuros, y "no tan grande que su colocación constituya probable incertidumbre $^{\prime 43}$. De una altura de 41 metros y once kilómetros aguas abajo del salto del Esla, el nuevo salto de Villalcampo debía terminarse en un plazo de cuatro años, pero en realidad se tardaron seis y se inauguró en 1949. Los principales problemas que provocaron el alargamiento de las obras estuvieron relacionados, a tenor de lo que se recogió en las actas de las reuniones de la comisión delegada, con los derivados de la situación general de España y con la dificultad de encontrar obreros adecuados y en cantidad suficiente, hasta el punto de plantearse una elevación de jornales para atraerlos ${ }^{44}$. Sin duda, a ello hay que sumar las dificultades que creó la guerra mundial para adquirir la maquinaria extranjera ${ }^{45}$. Sin embargo, la cuestión financiera se resolvió con facilidad, mediante una ampliación de capital de 120 millones de pesetas en 1943 que fue cubierta nada más salir al mercado, como explicó con satisfacción al consejo Ricardo Rubio ${ }^{46}$.

Resulta significativo que no exista una sola mención al problema de las tarifas eléctricas en las actas de las reuniones de la comisión delegada y del consejo de administración de Saltos del Duero entre 1936 y 1944. Podría pensarse que en parte se debiera a que Duero no era distribuidor sino productor, por lo que no le interesaban los precios finales, pero ello no es cierto por dos motivos: en primer lugar, porque distribuía en su mercado propio de Castilla, León y Extremadura y, en segundo, porque

Las escrituras de los convenios se encuentran en el Archivo de Iberdrola, Salto de Ricobayo, caja 198. Primero se firmó con Hidroeléctrica Española el 24 de marzo de 1944 y después con Unión Eléctrica Madrileña, Saltos del Alberche y Eléctrica de Castilla el 12 de mayo. Archivo de Iberdrola, CDSD, acta de 22/10/1942.

Archivo de Iberdrola, CDSD, acta de 1/09/1943 y 31/03/1944, y CASD, acta de 28/04/1944.

Pueyo (2007), p. 140, que cita como fuente una publicación de UNESA de 1947.

El Banco de Bilbao fue felicitado en el consejo de Duero por el papel desempeñado en la colocación de la emisión de acciones. Archivo de Iberdrola, CDSD, acta de 1/09/1943, y CASD, acta de 21/02/1944. 
los precios a los que le pagaban su producción las empresas distribuidoras se calculaban a partir de las tarifas que debían satisfacer a éstas los consumidores ${ }^{47}$. El asunto, en cambio, sí se mencionó en las discusiones para la fusión entre la Ibérica y Duero que dio lugar al nacimiento de Iberduero en el verano de 1944, ya que una de las diez ventajas que se apreciaron en la fusión fue precisamente el fortalecimiento de la posición de las empresas ante los abonados, "para lograr la regeneración de los precios de la energía, hoy envilecidos con relación al aumento que han experimentado otros productos" ${ }^{\prime 48}$.

La "pertinaz sequía" hizo su aparición en las actas de las reuniones de Saltos del Duero el 1 de septiembre de 1943 y ya no desapareció a lo largo del año que le quedaba de vida societaria hasta su fusión con la Ibérica ${ }^{49}$. El 16 de marzo de 1944, se elevó a la Jefatura de Industria de Vizcaya una comunicación en la que se indicaba que, en opinión de Duero, debía limitarse la producción de energía para no tener problemas de abastecimiento futuros, y por ello debían aplicarse restricciones al consumo cuanto antes. El 31 de marzo, se dice que "como la sequía general continúa pertinazmente, se han comenzado las restricciones de suministro de energía en Bilbao". Y en el verano estalla con toda su fuerza el problema de las restricciones, que ya no abandonarán a España definitivamente hasta más de un decenio después, con déficits que rondaron el $30 \%$ de la demanda total estimada en algunos años, como 1945 y $1949^{50}$. El 5 de julio de 1944, Ricardo Rubio informa al consejo:

“En el año actual ha sido preciso adelantar el periodo de restricciones en el consumo de energía, y, si las circunstancias no cambian, estas restricciones habrán de ser en término breve mucho más radicales. El aumento del consumo, la pertinaz sequía que pesa sobre toda la nación, la escasez de carbón para aprovisionamiento de térmicas y factores varios agudizan el problema y el Ministerio, ante la gravedad de la situación, viene adoptando medidas de rigor que las empresas tratan de observar escrupulosamente ${ }^{\prime \prime 51}$.

47 En junio de 1942, el Tribunal Arbitral del convenio al que habían sometido sus diferencias Saltos del Duero e Hidroeléctrica Ibérica determinó la fijación del precio medio a que la Ibérica debía pagar la energía suministrada por Duero: “Consistirá en dividir el importe total de la recaudación por la energía facturada a los abonados, multiplicada por 1,02. El 52 por 100 del precio así obtenido se aplicará al número de kwh entregados por Saltos del Duero en la central de Larrasquitu". Archivo de Iberdrola, CASD, acta de 22/10/1942. Archivo de Iberdrola, Salto de Ricobayo, caja 198: “Nota que elevan los Secretarios Generales de Saltos del Duero e Hidroeléctrica Ibérica a sus respectivos Presidentes sobre la fusión de las dos Sociedades", 12 de julio de 1944. Véase también Díaz Morlán (2006), p. 315.

49 Sería interesante averiguar si la conocidísima expresión franquista tuvo un origen empresarial, pero por el momento no estamos en disposición de asegurarlo. Quede la fecha de 1 de septiembre de 1943 para futuras comprobaciones.

$50 \quad$ Sudrià (1994), p. 333.

51 Archivo de Iberdrola, CASD, acta de 5/07/1944. 
Así pues, según este relato de los hechos, las empresas eléctricas sólo dejaron pasar un año largo desde el final de la guerra hasta que se plantearon la conveniencia de aumentar la producción ante las expectativas de incremento de la demanda, basadas fundamentalmente en las necesidades de nuevos proyectos industriales. Pero lo hicieron, lógicamente, dentro del marco fijado por el convenio de 1936, que daba a Duero la máxima potestad en materia de nuevas construcciones. Y Duero y el grupo hidroeléctrico tenían intereses opuestos, que llevaban al primero a desconfiar de las estimaciones de los segundos y a extremar la prudencia procurando no sobrevalorar la demanda futura, con el recuerdo de las preocupaciones que el exceso de oferta había provocado en los años treinta ${ }^{52}$. Cuando decidieron, en 1942, construir el salto de Villalcampo -que iba a doblar, no se olvide, la producción de la empresa, no encontraron ningún problema para cubrir la ampliación de capital requerida. Existía suficiente ahorro privado dispuesto a emplearse en proyectos hidroeléctricos, en parte debido, sin duda, a la falta de alternativas de inversión ${ }^{53}$.

En definitiva, los años prebélicos explican hechos relacionados con el sector eléctrico posteriores a la contienda, al menos en dos aspectos: la herencia de un convenio que define la actuación de las empresas y la herencia de una forma de pensar, cuya obsesión era la falta de demanda para cubrir toda la oferta disponible con la entrada en explotación del salto del Esla. Esa herencia pesó de manera determinante en el pensamiento de los directivos y consejeros de Duero, convertidos al acabar la guerra en responsables principales de la futura capacidad de producción eléctrica en España.

\section{Bibliografía}

ANTOLÍN, Francesca (1999): "Samuel Insull y Juan Urrutia, dos empresarios de principios de siglo. La formación de la gran empresa eléctrica española en un contexto comparativo", en GUTIÉRREZ, M. (coord.), Doctor Jordi Nadal. La industrialización y el desarrollo económico de España, vol. II, pp. 1356-1377.

Saltos del Duero no sería en esto una excepción. Una de las claves de la estrategia de gestión de Juan Urrutia en Hidroeléctrica Ibérica durante su primera etapa fue, en palabras de F. Antolín, la de "no aumentar la capacidad hasta no tener contratada nueva demanda", esto es, como dice la misma autora, adoptar "una política de expansión sumamente cautelosa". Antolín (1999).

53 Tampoco encontró problemas la Hidroeléctrica Ibérica en realizar en 1943 una ampliación de capital de sesenta millones de pesetas para financiar nuevas construcciones en el Pirineo. Antolín (2006), pp. 157-8. 
—(2006): "Hidroeléctrica Ibérica (1901-1944)", en GÓMEZ MENDOZA, Antonio; SAN ROMÁN, Elena y SUDRIÀ, Carles (coord.), Un siglo de luz. Historia empresarial de Iberdrola, Madrid, Iberdrola, pp. 131-191.

BARCIELA, Carlos, LÓPEZ, Inmaculada, MELGAREJO, Joaquín y MIRANDA, José Antonio (2001): La España de Franco (1939-1975). Economía, Madrid, Síntesis.

BARTOLOMÉ, Isabel (2007): La industria eléctrica en España (1890-1936), Madrid, Banco de España, Estudios de Historia Económica, ${ }^{\circ} 50$.

CARLSSON, Bo (1981): "Structure and performance in the West European steel industry: A historical perspective", en DE JONG, H.W. (ed.), The Structure of European Industry, The Hague/Boston/London, Martinus Nijhoff Publishers, pp. 115-140.

CATALÁN, Jordi (1994): “Industrialización difusa y desarrollo económico: el retroceso de 1939-1958", en NADAL, Jordi y CATALÁN, Jordi (eds.), La cara oculta de la industrialización española, Madrid, Alianza, pp. 369-396.

-(1995): La economía española y la segunda guerra mundial, Barcelona, Ariel.

DÍAZ MORLÁN, Pablo (1998): “El proceso de creación de Saltos del Duero (19171935)", en Revista de Historia Industrial, $\mathrm{n}^{\circ} 13$, pp. 181-198.

-(2002): Los Ybarra. Una dinastía de empresarios (1801-2001), Madrid, Marcial Pons.

—(2006): “Los Saltos del Duero (1918-1944)”, en Gómez Mendoza, A.; San Román, E. y Sudrià, C. (coor.), Un siglo de luz. Historia empresarial de Iberdrola, Madrid, Iberdrola, pp. 279-323.

GÓMEZ MENDOZA, Antonio (2000): “La tercera vía: entre la dictadura eléctrica y la autorregulación (1941/44)", en GÓMEZ MENDOZA, Antonio (ed.), De mitos y milagros. El Instituto Nacional de Autarquía (1941-1963), Barcelona, Fundación Duques de Soria y Universitat de Barcelona, pp. 69-84.

—(2007): "UNESA y la autorregulación de la industria eléctrica (1944-1973)", en GÓMEZ MENDOZA, Antonio; SUDRIÀ, Carles y PUEYO, Javier, Electra y el Estado. La intervención pública en la industria eléctrica bajo el franquismo, Pamplona, Civitas, vol. I, pp. 443-632.

PUEYO, Javier (2006): “La regulación económica de la industria eléctrica, 19391973", en GÓMEZ MENDOZA, Antonio; SAN ROMÁN, Elena y SUDRIÀ, Carles (coord.), Un siglo de luz. Historia empresarial de Iberdrola, Madrid, Iberdrola, pp. 349-381.

—(2007): “La regulación de la industria de producción y distribución de energía eléctrica en España, 1939-1972", en GÓMEZ MENDOZA, Antonio; SUDRIÀ, Carles y PUEYO, Javier, Electra y el Estado. La intervención pública en la industria eléctrica bajo el franquismo, Pamplona, Civitas, vol. I., pp. 65-439.

ROJO, Juan Carlos (2000): "Fernando María de Ybarra y de la Revilla (1875-1936)", en TORRES, E (dir.), Los cien empresarios españoles del siglo XX, Madrid, LID, pp. 234-238. 
SAN ROMÁN, Elena (comp.) (2006a): Cien años de Historia de Iberdrola. La historia económica, Madrid, Iberdrola.

—(2006b): “Hidroeléctrica Española: los difíciles años de la guerra civil”, en GÓMEZ MENDOZA, Antonio; SAN ROMÁN, Elena y SUDRIÀ, Carles (coord.), Un siglo de luz. Historia empresarial de Iberdrola, Madrid, Iberdrola, pp. 325-346.

SUDRIÀ, Carles (1990): “La industria eléctrica y el desarrollo económico de España” en GARCÍA DELGADO, José Luis (dir.), Electricidad y desarrollo económico: perspectiva histórica de un siglo, Oviedo, Hidroeléctrica del Cantábrico, pp. 147-184.

-(1994): "Un factor determinante: la energía", en NADAL, Jordi; CARRERAS, Albert y SUDRIÀ, Carles (comp.), La economía española en el siglo XX. Una perspectiva histórica, Barcelona, Ariel, pp. 313-363.

-(2001): "La economía española bajo el primer franquismo: la energía", ponencia presentada al VII Congreso de Historia Económica de la AEHE, Zaragoza, 19-21 de septiembre de 2001. Disponible en http://www.unizar.es/eueez/cahe/carlessudria.pdf.

-(2007): “El Estado y el sector eléctrico español bajo el franquismo: regulación y empresa pública", en GÓMEZ MENDOZA, Antonio; SUDRIÀ, Carles y PUEYO, Javier, Electra y el Estado. La intervención pública en la industria eléctrica bajo el franquismo, Pamplona, Civitas, vol. I, pp. 21-60.

TEDDE, Pedro y AUBANELL, Anna (2006): “Hidroeléctrica Española (1907-1936)", en GÓMEZ MENDOZA, Antonio; SAN ROMÁN, Elena y SUDRIÀ, Carles (coord.), Un siglo de luz. Historia empresarial de Iberdrola, Madrid, Iberdrola, pp. 193-277.

VALDALISO, Jesús María (2006). “Los orígenes de Hidroeléctrica Ibérica, Hidroeléctrica Española y Saltos del Duero", en GÓMEZ MENDOZA, Antonio; SAN ROMÁN, Elena y SUDRIÀ, Carles (coord.), Un siglo de luz. Historia empresarial de Iberdrola, Madrid, Iberdrola, pp. 97-129. 